\title{
2-D DOA Estimation of LFM Signals Based on Dechirping Algorithm and Uniform Circle Array
}

\author{
Kaibo CUI, Weiwei WU, Xi CHEN, Jingjian HUANG, Naichang YUAN \\ College of Electronic Science and Engineering, National University of Defense Technology, \\ Changsha, Hunan, 410073, China \\ 764608294@qq.com
}

Submitted July 31, 2016 /Accepted December 31, 2016

\begin{abstract}
Based on Dechirping algorithm and uniform circle array (UCA), a new 2-D direction of arrival (DOA) estimation algorithm of linear frequency modulation (LFM) signals is proposed in this paper. The algorithm uses the thought of Dechirping and regards the signal to be estimated which is received by the reference sensor as the reference signal and proceeds the difference frequency treatment with the signal received by each sensor. So the signal to be estimated becomes a single-frequency signal in each sensor. Then we transform the single-frequency signal to an isolated impulse through Fourier transform (FFT) and construct a new array data model based on the prominent parts of the impulse. Finally, we respectively use multiple signal classification (MUSIC) algorithm and rotational invariance technique (ESPRIT) algorithm to realize 2-D DOA estimation of LFM signals. The simulation results verify the effectiveness of the algorithm proposed.
\end{abstract}

\section{Keywords}

2-D DOA estimation, Dechirping algorithm, LFM signal, FFT, MUSIC algorithm, mode-space, ESPRIT algorithm

\section{Introduction}

Because of the advantages of UCA such as fewer array elements, the ability of 2-D DOA estimation, nonoriented fuzzy (uniform circular array is non-oriented fuzzy when the elements is an odd number greater than 5 or an even number greater than 8 [1]) etc., it has been widely used [2-7]. LFM signals have been widely used in sonar, radar and other detection equipments [8-12]. Especially for the development of imaging technology, LFM signals have become the main choice of the radar. So the DOA estimation of LFM signals based on UCA becomes an important issue. However, as the LFM signal belongs to a typical non-stationary signal, the traditional subspace algorithms which are based on the stationary signals cannot be applied to such condition. With the development of signal processing technology, people have developed a series of DOA estimation algorithms suitable for wideband signals [13-19]. In [13], the author uses a triangular array to realize DOA estimation of broadband LFM signals. There is no need for such algorithm to estimate the signal parameters and solve the signal spectrum. In [14], the author uses a space-time extended MUSIC estimation algorithm to realize DOA estimation of wideband signals. The algorithm shows a good ability to estimate a number of sources that exceed the number of sensors in the array. In [15-19], people use the sparse matrix theory to realize DOA estimation of wideband signals. Since Amin introduces the time-frequency analysis tool to DOA estimation field in 1999 [20], [21], people also have developed a series of DOA estimation algorithms based on the time-frequency analysis tool. In [22-24], the authors proceed DOA estimation by using Wigner-Ville distribution (WVD). But the calculation of WVD is very complex and the crossterms seriously affect the estimation accuracy in the case of multiple signals. In [25], [26], the authors study the DOA estimation algorithm based on short-time Fourier transform (STFT). These kinds of algorithms avoid cross terms interference of WVD, but it is difficult to select an appropriate time-frequency point. Especially, the irreconcilable conflicts between the STFT window length (the calculation complexity of the algorithm) and the estimate accuracy limit further development of such algorithm. In [27-32], the authors study DOA estimation of LFM signals based on fractional Fourier transform (FRFT). They construct a novel array data mode in the fractional Fourier transform domain (FRFD) based on a fact that LFM signals have the energy concentrated performance in the FRFD and then use MUSIC algorithm to obtain the 2-D DOA estimation of multiple LFM sources. But in their papers, they just discuss the linear and rectangular array model. It is necessary to increase the number of array elements to achieve high accuracy, which is difficult to realize. Also, FRFT is difficult to understand and project implementation. So these kinds of algorithms have not been widely used.

This paper studies 2-D DOA estimation algorithm of LFM signals based on Dechirping algorithm and UCA. Firstly, we use the Dechirping algorithm to process LFM signals received by each array element and transform signals from time domain to frequency domain using Fourier 
transform (FFT). Then, we construct a new array data model through extracting the prominent parts of the impulse in the frequency domain. Finally, we realize 2-D DOA estimation of LFM signals using MUSIC algorithm and ESPRIT algorithm respectively. For the LFM signals, the time-frequency analysis tools are very suitable to process them [33]. So the main DOA estimation algorithms of LFM signals are based on the time-frequency analysis tools. Dechirping algorithm can reduce the quantity of data [34], so we choose it to estimate DOA of LFM signals in this paper. Also in the method proposed in this paper the cross-term interference does not exist which exists in the DOA algorithms based on WVD. It also does not have the puzzle of selecting the appropriate window function compared to the DOA algorithms based on STFT. The algorithm also does not require selecting the correct time frequency points compared to the DOA algorithms based on WVD and STFT. Unlike the existing methods which are based on more computationally expensive approach, the proposed one is significantly more efficient in terms of computational complexity. The algorithm also uses the relatively simple FFT operator and has the capability of estimating DOA of multiple LFM signals simultaneously with a high precision which are superior to the DOA algorithm based on FRFT. So the proposed algorithm is prone to be easily realized in engineering. The simulation results showed the good performance of the algorithm proposed by this paper.

The paper is organized as follows. In Sec. 2, we introduce the new array data model of LFM signals based on Dechirping algorithm and we proceed detailed derivation in this part. In Sec. 3, we realize 2-D DOA estimation of LFM signals using MUSIC algorithm and ESPRIT algorithm respectively. For ESPRIT algorithm, we also study the mode-space algorithm. In Sec. 4, we proceed the numerical simulation on the algorithm proposed by this paper. Finally, Section 5 concludes the paper.

\section{The Novel UCA Data Model of LFM Signals Based on Dechirping Algorithm}

The UCA model with $N$ sensors is shown in Fig. 1. The UCA radius is $r$ and $A_{1}, A_{2}, \ldots, A_{N}$ are elements of the UCA separately. Without loss of generality, we can set the angle between $A_{1}$ and $X$ axis to be $w_{0}$ and the angle between the array elements is $w$, so $w=2 \pi / N$. The angle between the far-field incident wave and $Z$ axis is $\beta$, which is called the elevation angle. The angle between the projection of the incident wave in $X O Y$ plane and $X$ axis is $\alpha$, which is called the azimuth angle.

If there are $M$ LFM signals from far-field, the output of the $n$th element can be written as:

$$
x_{n}(t)=\sum_{m=1}^{M} s_{m}\left(t-\tau_{n m}\right)+n_{\mathrm{n}}(t), n=1,2, \ldots, N,
$$

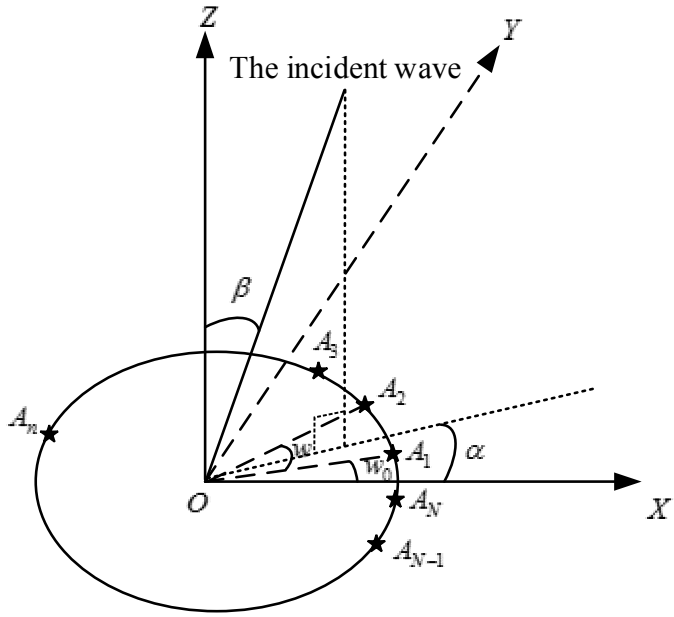

Fig. 1. The uniform circular array model.

$$
s_{m}(t)=\exp \left[\mathrm{j} \pi\left(2 f_{m} t+\gamma_{m} t\right)\right] .
$$

In (1) and (2), $n_{\mathrm{n}}(t)$ is the noise, $s_{m}(t)$ is the $m$ th LFM sig$\mathrm{nal}, f_{m}$ is the central frequency of the $m$ th LFM signal, $\gamma_{m}$ is the modulated frequency of the $m$ th LFM signal, $\tau_{n m}$ is the time delay of the $m$ th LFM signal on the $n$th element respect to the reference element. If we regard $O$ as a virtual reference element and the speed of light is $c$, the time delay caused by the wave path difference for each element respect to the reference element is:

$$
\tau_{n m}(t)=\frac{r}{c} \sin \left(\theta_{\mathrm{e} m}\right) \cos \left(\theta_{\mathrm{a} m}-\frac{2 \pi(n-1)}{N}\right) .
$$

In (3), $\theta_{\mathrm{a} m}$ is the azimuth angle of the $m$ th LFM signal and $\theta_{\mathrm{e} m}$ is the elevation angle of the $m$ th LFM signal.

In this paper, we assume that the modulated frequencies of LFM signals are not identical, namely:

$$
\gamma_{i} \neq \gamma_{j}, i \neq j, i, j \in 1,2, \ldots, M .
$$

Dechirping algorithm is a kind of time-domain transform technique used in high-resolution radar, which can greatly reduce the amount of data, so it has been widely used in the imaging field. Firstly, we construct a LFM signal whose central frequency and modulated frequency are the same as the original LFM signal. Then, we process difference frequency treatment between the original signal and the reference signal. Finally, we can get a single-frequency signal. For the LFM signals received by each array element, we use the $i$ th LFM signal received by the reference element to carry through Dechirping process. So we can get:

$$
y_{n}(t)=x_{n}(t) * s_{i}^{*}(t)=\sum_{m=1}^{M} z_{n m}(t)
$$

$z_{n m}(t)=$

$\exp \left\{\mathrm{j} \pi\left[\left(\gamma_{m}-\gamma_{i}\right) t^{2}+2\left(f_{m}-f_{i}-\gamma_{m} \tau_{n m}\right) t+\gamma_{m} \tau_{n m}^{2}-2 f_{m} \tau_{n m}\right]\right\}$.

Due to the different modulated frequency of all LFM signals, $z_{n m}(t)$ is a single-frequency signal just when $m=i$, otherwise $z_{n m}(t)$ is a LFM signal. So the received signals of each element are the superposition between $(M-1)$ LFM 
signals and a single-frequency signal after Dechirping process.

For (5) and (6), we can execute FFT on both ends of the equal sign to get the frequency-domain expression.

$$
\begin{aligned}
Y_{n}(\omega)= & 2 \pi \exp \left[j \pi\left(\gamma_{i} \tau_{n i}{ }^{2}-2 f_{i} \tau_{n i}\right)\right] \delta\left(\omega+2 \pi \gamma_{i} \tau_{n i}\right)+ \\
& \sum_{m=1, m \neq i}^{M} \frac{1}{\sqrt{\left|Q_{m}\right|}} \exp \left[j \pi\left(C_{n m}+\frac{1}{4}\right)\right] \exp \left[-j \pi \frac{\left(\frac{\omega}{2 \pi}-B_{n m}\right)^{2}}{Q_{m}}\right] .
\end{aligned}
$$

In (7), $\delta$ is the impulse function, $Q_{m}=\gamma_{m}-\gamma_{i}$, $B_{n m}=f_{m}-f_{i}-\gamma_{m} \tau_{n m}, C_{n m}=\gamma_{m} \tau_{n m}{ }^{2}-2 f_{m} \tau_{n m}$.

Since each LFM signal owns a certain bandwidth, the result of (7) is an isolated impulse point when $\omega=-2 \pi \gamma_{i} \tau_{n i}$. So we can regard this point as the output of the array, namely:

$$
\begin{aligned}
X_{n} & =\left.Y_{n}(\omega)\right|_{\omega=-2 \pi \gamma_{i} \tau_{n i}} \\
& =2 \pi \exp \left[\mathrm{j} \pi\left(\gamma_{i} \tau_{n i}{ }^{2}-2 f_{i} \tau_{n i}\right)\right]+2 \pi \sum_{m=1, m \neq i}^{M} D_{n m}, \\
D_{n m} & =\frac{1}{2 \pi \sqrt{\left|Q_{m}\right|}} \exp \left[\mathrm{j} \pi\left(C_{n m}+\gamma_{i} \tau_{n i}+B_{n m}+\frac{1}{4}\right)\right] .
\end{aligned}
$$

For (8), we only take the prominent parts of the isolated impulse for each LFM signals.

$$
\begin{gathered}
X_{n i}=2 \pi A_{n i}, \\
A_{n i}=\exp \left[\mathrm{j} \pi\left(\gamma_{i} \tau_{n i}{ }^{2}-2 f_{i} \tau_{n i}\right)\right] .
\end{gathered}
$$

The second term of $A_{n i}$ is very small, so we generally ignore it in practice, namely:

$$
A_{n i} \approx \exp \left[-\mathrm{j} 2 \pi f_{i} \tau_{n i}\right] .
$$

We can let $i$ traverse from 1 to $M$ and get $X_{n i}$ through the above method. In this way, we can get a new array data model.

$$
\left\{\begin{array}{l}
\mathbf{X}=\mathbf{A S}+\mathbf{N} \\
\mathbf{X}=\left[\mathbf{X}_{1}, \ldots, \mathbf{X}_{N}\right]^{\mathrm{T}} \\
\mathbf{X}_{n}=\left[X_{n 1}, \ldots, X_{n M}\right] \\
\mathbf{A}=\left[\mathbf{A}_{1}, \ldots, \mathbf{A}_{M}\right] \\
\mathbf{A}_{m}=\left[A_{1 m}, \ldots A_{N m}\right]^{\mathrm{T}} \\
\mathbf{S}=\operatorname{diag}\{\overbrace{2 \pi, \ldots, 2 \pi}^{M}\}
\end{array}\right.
$$

\section{2-D DOA Estimation Algorithm Based on Dechirping Algorithm}

\subsection{Dechirping-MUSIC Algorithm}

From (13), we can get the correlation matrix of the array output [35].

$$
\mathbf{R}_{\mathbf{X}}=E\left[\mathbf{X} \mathbf{X}^{\mathrm{H}}\right]=\mathbf{A} \mathbf{R}_{\mathbf{S}} \mathbf{A}^{\mathrm{H}}+\sigma^{2} \mathbf{I} .
$$

In (14), $\mathbf{R}_{\mathrm{S}}$ is the correlation matrix of the LFM signals, $\sigma^{2}$ is the power of Gaussian white noise, $\mathbf{I}$ is the unit matrix. Since the signal and noise are independent, $\mathbf{R}_{\mathrm{X}}$ can be decomposed into two parts: signal and noise. So, we can proceed features decomposition and get the following expression.

$$
\mathbf{R}_{\mathbf{X}}=\mathbf{U}_{\mathbf{S}} \sum_{\mathrm{s}} \mathbf{U}_{\mathbf{S}}{ }^{\mathrm{H}}+\mathbf{U}_{\mathbf{N}} \sum_{\mathrm{N}} \mathbf{U}_{\mathbf{N}}{ }^{\mathrm{H}} .
$$

In (15), $\mathbf{U}_{\mathrm{S}}$ and $\mathbf{U}_{\mathrm{N}}$ respectively are signal subspace and noise subspace. So we can construct the MUSIC spatial spectrum [31] as follows.

$$
P\left(\theta_{\mathrm{a} m}, \theta_{\mathrm{e} m}\right)=\frac{1}{\mathbf{A}_{m}{ }^{\mathrm{H}}\left(\theta_{\mathrm{a} m}, \theta_{\mathrm{e} m}\right) \mathbf{U}_{\mathbf{N}} \mathbf{U}_{\mathbf{N}}{ }^{\mathrm{H}} \mathbf{A}_{m}\left(\theta_{\mathrm{a} m}, \theta_{\mathrm{e} m}\right)} .
$$

We can proceed two-dimensional search based on (16) and find the angle $\left(\theta_{\mathrm{a} m}, \theta_{\mathrm{e} m}\right)$ when its value is maximum. $\left(\theta_{\mathrm{a} m}, \theta_{\mathrm{e} m}\right)$ is the DOA of the $m$ th LFM signal.

From (16), we can also see that $\mathbf{A}_{m}\left(\theta_{\mathrm{a} m}, \theta_{\mathrm{e} m}\right)$ is different for different LFM signals, so in the case of multiple signals, the Dechirping-MUSIC algorithm needs to proceed the two-dimensional search repeatedly, which causes huge computation. So it is necessary to research the algorithm based on Dechirping algorithm and ESPRIT algorithm.

\subsection{Dechirping-ESPRIT Algorithm}

ESPRIT algorithm needs rotational invariance of the array [36], [37]. But the circular array does not have this structural characteristic, so we use mode-space algorithm firstly in this paper. Mode-space algorithm is a kind of spectral estimation algorithm for the UCA [38], [39] and its central idea is to equalize a UCA to a virtual uniform linear array (ULA) through matrix transformation so that the DOA estimation algorithm based on the ULA can be applied to a UCA. We ignore the details of the mode-space algorithm in the main body and show the results as (17). The details of UCA equalization to ULA are included in Appendix: The derivation of (17). 


$$
\begin{aligned}
& \left\{\begin{array}{l}
\mathbf{Y}=\mathbf{T X}=\mathbf{A}_{c} \mathbf{S}+\mathbf{N} \\
\mathbf{A}_{c}=\left[\begin{array}{ccc}
J_{-K}\left(-\beta_{1}\right) \exp \left(-\mathrm{j} K \theta_{\mathrm{e} 1}\right) & \cdots & J_{-K}\left(-\beta_{M}\right) \exp \left[-\mathrm{j} K \theta_{\mathrm{e} M}\right] \\
\cdots & \ddots & \vdots \\
J_{K}\left(-\beta_{1}\right) \exp \left[\mathrm{j} K \theta_{\mathrm{e} 1}\right] & \cdots & J_{K}\left(-\beta_{M}\right) \exp \left[\mathrm{j} K \theta_{\mathrm{e} M}\right]
\end{array}\right]
\end{array}\right. \\
& \left\{\mathbf{T}=\frac{1}{N} \mathbf{J}^{-1} \mathbf{F}^{\mathrm{H}}\right. \\
& \mathbf{F}=\left[\begin{array}{llll}
\mathbf{w}_{-K} & \mathbf{w}_{-K+1} & \cdots & \mathbf{w}_{K}
\end{array}\right]
\end{aligned}
$$

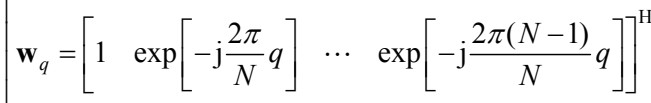

$$
\begin{aligned}
& \mathbf{J}=\operatorname{diag}\left\{\mathbf{j}^{-K}, \ldots, \mathbf{j}^{K}\right\}
\end{aligned}
$$

In (17), $\mathbf{Y}$ is the output of the virtual ULA, $\mathbf{T}$ is the transformation matrix, $\mathbf{A}_{\mathrm{c}}$ is the manifold matrix of the virtual linear array, $\mathbf{F}$ is the spatial discrete Fourier transform (DFT) transformation matrix, $J_{m}(x)$ is the order $m$ of Bessel function, $\beta_{m}$ is a factor related with the incoming wave parameters. $K$ is the maximum phase mode excited by the UCA, so the number of phase mode excited is $2 K+1$.

$$
\begin{gathered}
\beta_{m}=\frac{2 \pi r f_{m}}{c} \sin \theta_{\mathrm{e} m} . \\
K=\left\lfloor\min \left[\frac{2 \pi r f_{m}}{c}\right]\right\rfloor .
\end{gathered}
$$

In (19), min \lfloor\rfloor expresses the minimum, \lfloor\rfloor is the rounded down symbol.

In this way, the UCA is converted to be a ULA whose element number is $2 K+1$.

As the parameters of LFM signals are different, so:

$$
\beta_{i} \neq \beta_{j}, i \neq j, i, j=1,2, \ldots, M \text {. }
$$

Based on (20), we can see that $\mathbf{A}_{c}$ is not a general manifold matrix of the ULA. So we cannot simply divide the array into several sub-arrays and use ESPRIT algorithm. Bessel functions have the recursive nature of the order, namely:

$$
J_{m-1}(x)+J_{m+1}(x)=\frac{2 m}{x} J_{m}(x) .
$$

We can get the following expressions from (17) and (21).

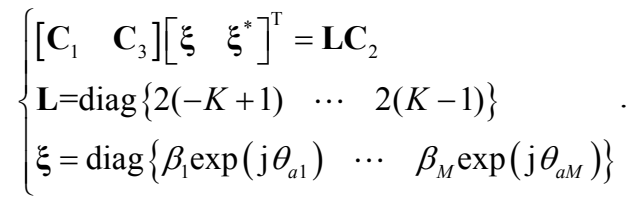

In (22), $\mathbf{C}_{1}$ is a $(2 K-1) \times M$ sub-array cut out at the beginning of the first line of $\mathbf{A}_{\mathrm{c}}, \mathbf{C}_{2}$ is a $(2 K-1) \times M$ subarray cut out at the beginning of the second line of $\mathbf{A}_{c}$, and $\mathbf{C}_{3}$ is a $(2 K-1) \times M$ sub-array cut out at the beginning of the third line of $\mathbf{A}_{\mathrm{c}}$.

Because $\mathbf{A}_{\mathrm{c}}$ is the manifold matrix of the virtual ULA, so $\mathbf{C}_{1}, \mathbf{C}_{2}$ and $\mathbf{C}_{3}$ separately are the manifold matrix of the three sub-array, which correspond to their own signal subspace, so (22) can also be written as:

$$
\left\{\begin{array}{l}
{\left[\begin{array}{ll}
\mathbf{U}_{\mathrm{S}_{1}} & \mathbf{U}_{\mathrm{S}_{3}}
\end{array}\right] \boldsymbol{\Psi}=\mathbf{L} \mathbf{U}_{\mathbf{S}_{2}}} \\
\boldsymbol{\Psi}=\left[\begin{array}{ll}
\xi & \xi^{*}
\end{array}\right]^{\mathrm{T}}
\end{array}\right.
$$

In (23), $\mathbf{U}_{\mathrm{S}_{1}}, \mathbf{U}_{\mathrm{S}_{2}}$ and $\mathbf{U}_{\mathrm{S}_{3}}$ separately are the signal subspaces of the three sub-arrays.

We can use the least square (LS) method to solve the above equation and the result is:

$$
\boldsymbol{\psi}_{\mathrm{LS}}=\left[\begin{array}{ll}
\boldsymbol{\psi}_{\mathrm{LS} 1} & \boldsymbol{\psi}_{\mathrm{LS} 2}
\end{array}\right]^{\mathrm{T}}=\left[\begin{array}{ll}
\mathbf{U}_{\mathrm{S}_{1}} & \mathbf{U}_{\mathrm{S}_{3}}
\end{array}\right]^{+} \mathbf{L U}_{\mathrm{S}_{2}} .
$$

In (24), ${ }^{+}$represents the generalized inverse matrix. $\psi_{\mathrm{LS} 1}$ is the first $M$ rows data of $\psi_{\mathrm{LS}}$ and $\psi_{\mathrm{LS} 2}$ is the second $M$ rows data of $\psi_{\text {LS. }}$ We can separately proceed features decomposition for $\psi_{\mathrm{LS} 1}$ and $\psi_{\mathrm{LS} 2}$. The eigenvalues of them respectively are $\lambda_{1 m}(m=1, \ldots, M)$ and $\lambda_{2 m}(m=1, \ldots, M)$, so the DOA of the $m$ th LFM signal is:

$$
\left\{\begin{array}{l}
\theta_{\mathrm{a} m}=\arg \left(\lambda_{1 m} / \lambda_{2 m}\right) / 2 \\
\theta_{\mathrm{e} m}=\arcsin \left(\frac{\operatorname{real}\left(\lambda_{1 m}\right) c}{2 \pi r f_{m} \cos \theta_{\mathrm{a} m}}\right)
\end{array} .\right.
$$

In (25), $\arg ()$ represents a phase angle function of a plural and real () means to take the real part of a plural.

We can see from (25) that the Dechirping-ESPRIT algorithm does not need to proceed eigen decomposition repeatedly in the case of multiple signals.

For the readers' convenience, the step by step procedure of the proposed algorithm is given in Tab. 1 .

If there are $M$ LFM signals arriving at a UCA with $N$ sensors and the sampling numbers are $L$ for each signal. We can analyze the computational complexity of the algorithm based on the procedure of the algorithm in Tab. 1 . For the DOA estimation algorithms based on timefrequency analysis tools, the differences of the computational complexity are mainly concentrated on the process of

1) Select the $i$ th LFM signal as the reference signal to proceed Dechirping process.

2) Execute FFT on the Dechirping results and get the prominent parts of the isolated impulse.

3) Let $i$ traverse from 1 to $M$ and repeat (1) - (2) to get the novel array data model. For Dechirping-MUSIC algorithm, proceed (4). For Dechirping-ESPRIT algorithm, proceed (5)-(7).

4) Proceed the two-dimensional search according to (16) and get the DOA estimation.

5) Proceed the mode-space transformation based on (17) and get the new manifold matrix.

6) Construct the rotational invariant equation according to (23) and get $\psi_{\mathrm{LS}}$ according to (24).

7) Proceed eigen decomposition for $\psi_{\mathrm{LS}}$ and obtain the DOA estimation based on (25).

Tab. 1. The complete procedure of the algorithm proposed. 
establishing the new array model. So the algorithm computational complexity in this paper is the computational complexity of establishing the array model. Based on the procedure of the algorithm in Tab. 1, $M$ LFM signals need to be processed by Dechirping algorithm in each sensor firstly and the computation is $M N L$. Then, the Dechirping results are transformed from time domain to frequency domain using FFT whose computation is $M N L \log _{2} L$. Finally, we can construct a new array data model through extracting the prominent parts of the impulse in the frequency domain and the computation is $M N$. So the computational complexity of the proposed algorithm is $O\left(M N L \log _{2} L\right)$. For the DOA estimation algorithms based on WVD [20-24], the computational complexity is $O\left(M N^{2} L^{2} \log _{2} L\right)$. For the DOA estimation algorithms based on STFT [25], [26], the computational complexity is $O\left(M N L^{2} \log _{2} L\right)$. For the DOA estimation algorithms based on FRFT [27-32], the computational complexity is $O\left(N L+M N^{2}\right)$. So the computational complexity of the proposed algorithm achieves a low level contrast to the other DOA estimation algorithms based on time-frequency analysis tools, which is prone to realization in engineering.

\section{Numerical Simulation}

We proceed the numerical simulation of the proposed algorithm in this paper and use a UCA with sixteen sensors

\begin{tabular}{|c|c|c|c|}
\hline $\begin{array}{c}\text { Signal } \\
\text { number }\end{array}$ & $\begin{array}{c}\text { Frequency } \\
{[\mathrm{Hz}]}\end{array}$ & $\begin{array}{c}\text { Modulated } \\
\text { frequency }[\mathrm{Hz} / \mathrm{s}]\end{array}$ & $\begin{array}{c}\text { Sampling } \\
\text { rate }[\mathrm{Hz}]\end{array}$ \\
\hline 1 & $10 \times 10^{8}$ & $6 \times 10^{12}$ & $10^{8}$ \\
\hline 2 & $8 \times 10^{8}$ & $-2 \times 10^{12}$ & $10^{8}$ \\
\hline 3 & $9 \times 10^{8}$ & $3 \times 10^{12}$ & $10^{8}$ \\
\hline $\begin{array}{c}\text { Signal } \\
\text { number }\end{array}$ & $\begin{array}{c}\text { Snapshot } \\
\text { numbers }\end{array}$ & SNR [dB] & DOA [ $\left.{ }^{\circ}\right]$ \\
\hline 1 & 300 & 20 & $(-25,-35)$ \\
\hline 2 & 300 & 20 & $(25,-15)$ \\
\hline 3 & 300 & 20 & $(35,15)$ \\
\hline
\end{tabular}

Tab. 2. The parameters of three LFM signals.

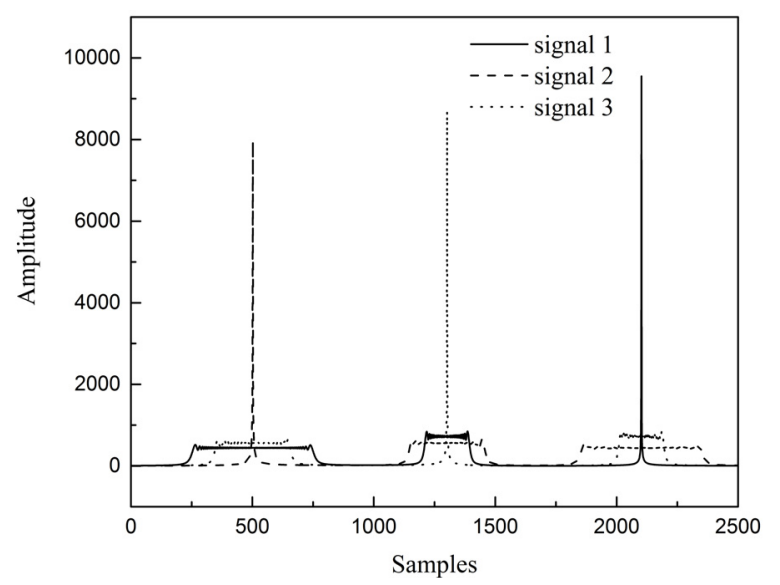

Fig. 2. The results of the reference element after Dechirping process and FFT. whose radius is $0.4 \mathrm{~m}$. There are three far-field LFM signals whose parameters are shown in Tab. 2 .

Firstly, the LFM signals received by each element are processed by the Dechirping algorithm and the frequency domain result of the reference element is shown in Fig. 2.

As can be seen from the figure, the three LFM signals correspond to the three isolated impulse points in the frequency domain after Dechirping process. We can take the prominent parts of the isolated impulse for each array element to get the new array output matrix.

\subsection{Dechirping-MUSIC Algorithm Simulation}

We use the Dechirping-MUSIC algorithm to estimate DOA of the three LFM signals and the MUSIC spectrum is shown in Fig. 3. In order to facilitate the observation, the MUSIC spectrum of these three signals is displayed on a map, which means that the value of the azimuth angle and elevation angle is changed every three points.

We proceed the two-dimensional search on the MUSIC spectrum and the search range are all from $-90^{\circ}$ to $90^{\circ}$ in the azimuth and elevation direction. The search step is $0.01^{\circ}$. We can get the estimates of DOA by searching the peak point which respectively are: $\left(-25.02^{\circ},-35.01^{\circ}\right)$, $\left(25.02^{\circ},-14.99^{\circ}\right)$, and $\left(35.02^{\circ}, 15.01^{\circ}\right)$. The estimates match with the DOA set in Tab. 2.

In order to further examine the performance of the algorithm, we also analyze the relationship between the root mean square error (RMSE) of DOA estimation and signal to noise ratio (SNR) of LFM signals. In addition to an incremental SNR of LFM signals, the other parameters remain unchanged. In order to reduce the effect of random errors, we proceed a total of 1000 times Monte Carlo simulation and the results are shown in Fig. 4.

In Fig. 4(a) there is the RMSE of azimuth angle along with SNR and in Fig. 4 (b) there is the RMSE of elevation angle along with SNR. For comparison purpose, the Cramer-Rao lower bound (CRLB) [40-44] is also presented in Fig. 4. As can be seen from the figure, the RMSE

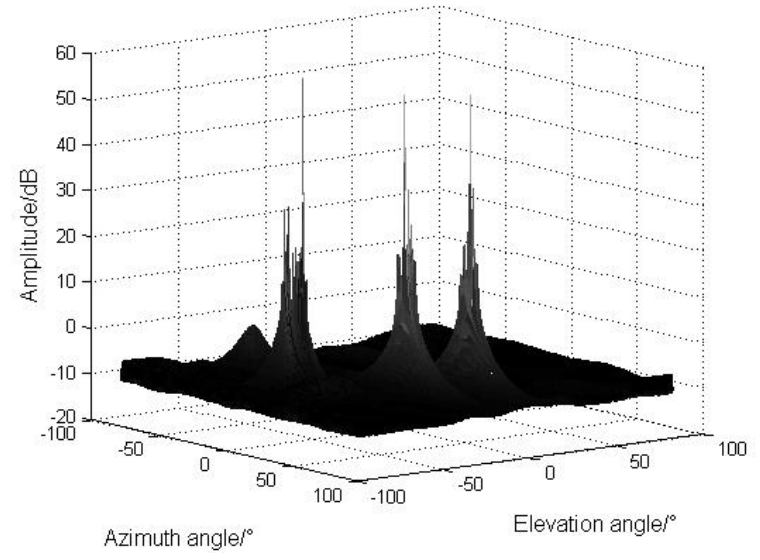

Fig. 3. The Dechirping-MUSIC spectrum of these three LFM signals. 


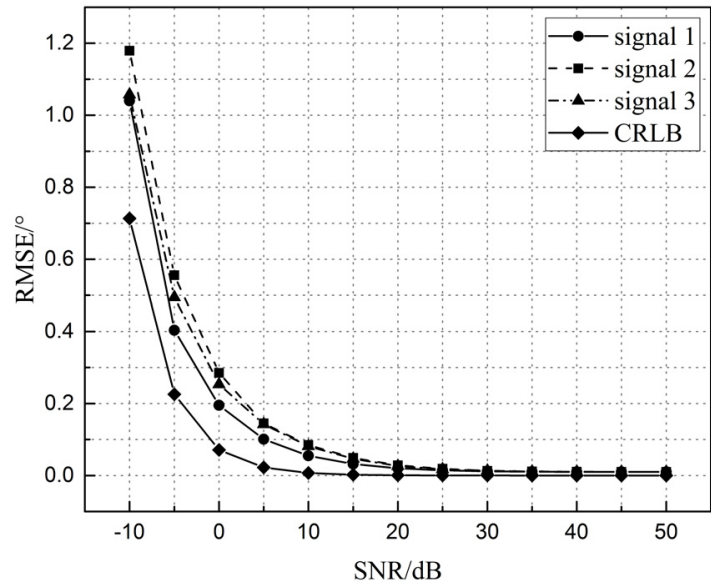

(a) Azimuth angle

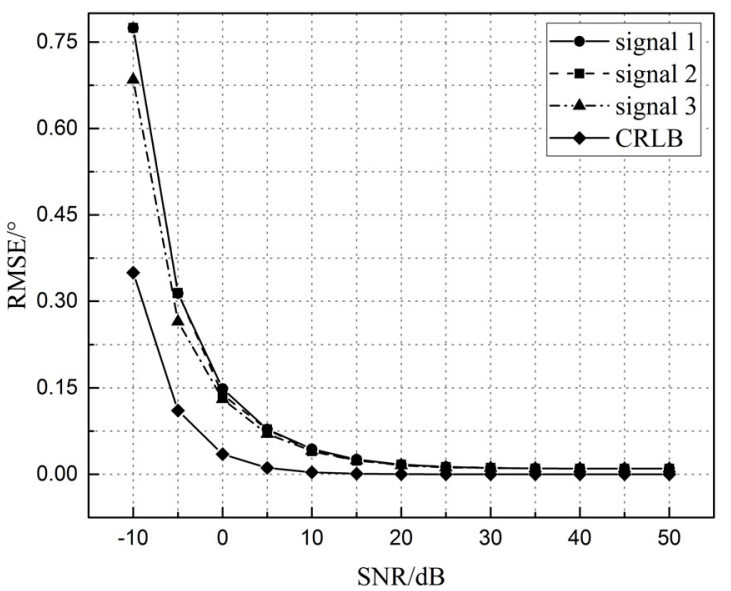

(b) Elevation angle

Fig. 4. RMSE of DOA estimation along with SNR using Dechirping-MUSIC algorithm.

of DOA estimations decrease rapidly as the SNR of signals increase and achieve the convergence condition (The RMSE is infinitely close to zero) ultimately. In the case of low SNR (less than $0 \mathrm{~dB}$ ), the algorithm still has a good estimation performance. For a UCA, it has isotropy for the waves of different direction in theory, however we use the approximation in (12) when constructing the new array data model, so the DOA estimation performance for the waves of different direction are not the same. The simulation results are in line with expectations.

\subsection{Dechirping-ESPRIT Algorithm Simulation}

We use the Dechirping-ESPRIT algorithm to estimate DOA of the three LFM signals. According to (19), we can get that the value of $K$ is 6 , which means the number of phase mode excited is 13 , so we can equalize this UCA to a ULA with 13 elements based on (17). Then following the steps 6) and 7), we can obtain the estimates of DOA for these three LFM signals, which are: $\left(-25.28^{\circ},-34.96^{\circ}\right)$, $\left(25.19^{\circ},-14.94^{\circ}\right)$, and $\left(35.26^{\circ}, 14.93^{\circ}\right)$. The estimates match with the angle set in Tab. 2.

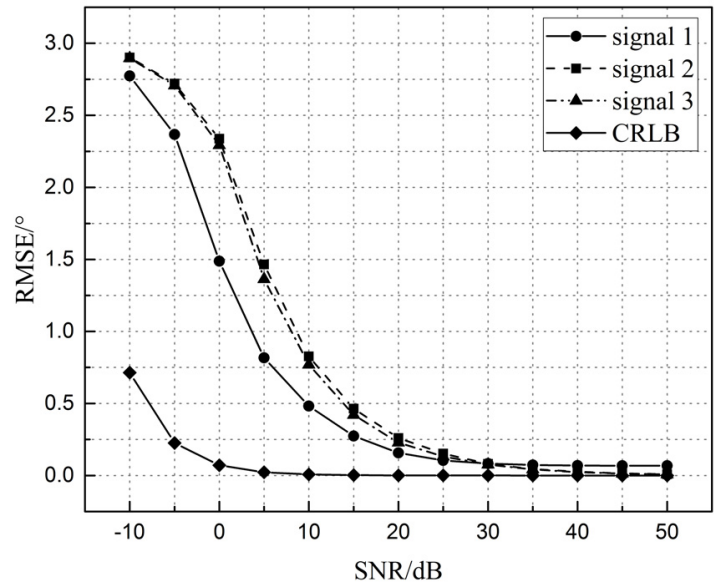

(a) Azimuth angle

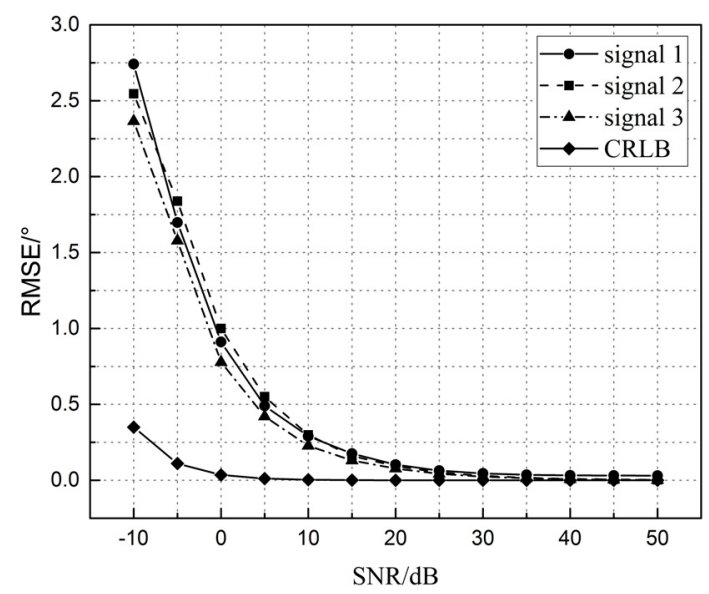

(b) Elevation angle

Fig. 5. RMSE of DOA estimation along with SNR using Dechirping-ESPRIT algorithm.

This paper also simulates the relationship between RMSE of DOA estimation of Dechirping-ESPRIT algorithm and SNR of these three LFM signals. In addition to an incremental SNR, the other signal parameters remain unchanged. We proceed a total of 1000 times Monte Carlo simulation and the results are shown in Fig. 5.

In Fig. 5(a) there is the RMSE of azimuth angle along with SNR and in Fig. 5(b) there is the RMSE of elevation angle along with SNR. As can be seen from the figure, the RMSE of DOA estimations decrease rapidly as the SNR of signals increase and achieve the convergence condition ultimately which are similar to the Dechirping-MUSIC algorithm. Compared with the Dechirping-MUSIC algorithm, the RMSE of DOA estimation of DechirpingESPRIT algorithm is larger, which means the estimation precision is lower. This is due to the inherent high precision of MUSIC algorithm [44]. But the advantage of Dechirping-ESPRIT algorithm is that the calculation amount is less. Especially in the case of multiple signals, the Dechirping-ESPRIT algorithm does not need to proceed eigen decomposition repeatedly, which reduces the computational complexity greatly. When using DechirpingESPRIT algorithm, we use the approximation not only 
when constructing the new array data model but also when proceeding mode-space conversion in (17), so the DOA estimation performance for the waves of different direction are not the same and the differences are more obvious compared with Dechirping-MUSIC algorithm according to Fig. 4 and Fig. 5. The simulation results are in line with expectations.

\subsection{Algorithm Comparison Simulation}

In order to further examine the performance of the algorithm, we proceed the comparison simulation between WVD-MUSIC algorithm in [21], WVD-ESPRIT algorithm in [24], STFT-ESPRIT algorithm in [25], STFT-MUSIC algorithm in [26], FRFT-ESPRIT algorithm in [27], FRFTMUSIC algorithm in [29] and the algorithm proposed by this paper. We select signal 1 in Tab. 2 as the incoming wave signal and the parameters of uniform circle array are unchanged. We proceed a total of 1000 times Monte Carlo simulation and the results are shown in Fig. 6.

In Fig. 6(a) there is the RMSE of azimuth angle along with SNR based on MUSIC algorithm, (b) is the RMSE of elevation angle along with SNR based on MUSIC algorithm, (c) is the RMSE of azimuth angle along with SNR based on ESPRIT algorithm, (d) is the RMSE of elevation angle along with SNR based on ESPRIT algorithm. We can see from Fig. 6 that the estimation precision of four kinds of DOA estimation algorithm is every high and they all can achieve the convergence condition finally. By contrast, the algorithm based on WVD owns the highest estimation precision, followed by the algorithm based on STFT, the algorithm proposed in this paper and the algorithm based on FRFT. Meanwhile, the estimation precision differences of these four kinds of algorithm are not big. Especially in high SNR situation, the estimation precision is almost the same. But based on the above analysis, the algorithm proposed in this paper owns the lowest computational complexity and uses the relatively simple FFT operator. In addition, when constructing the new array data model of LFM signals, the algorithm proposed in this paper extracts only the prominent parts of the impulse.

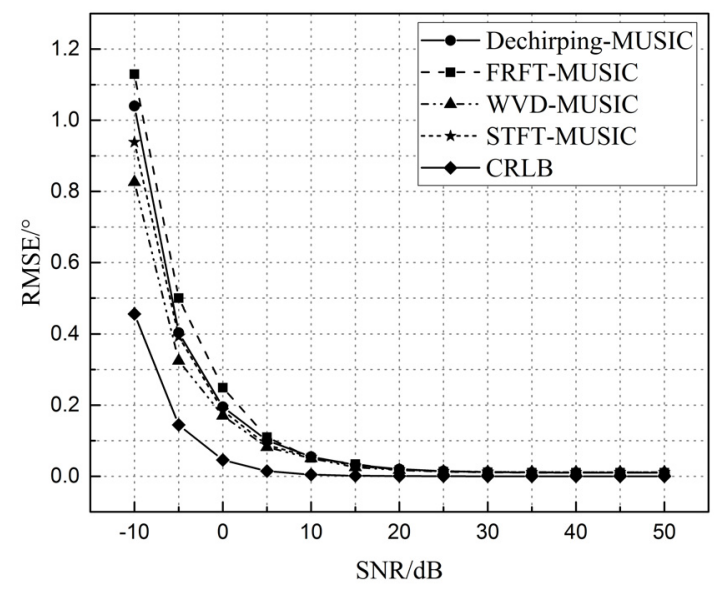

(a) Azimuth angle based on MUSIC algorithm

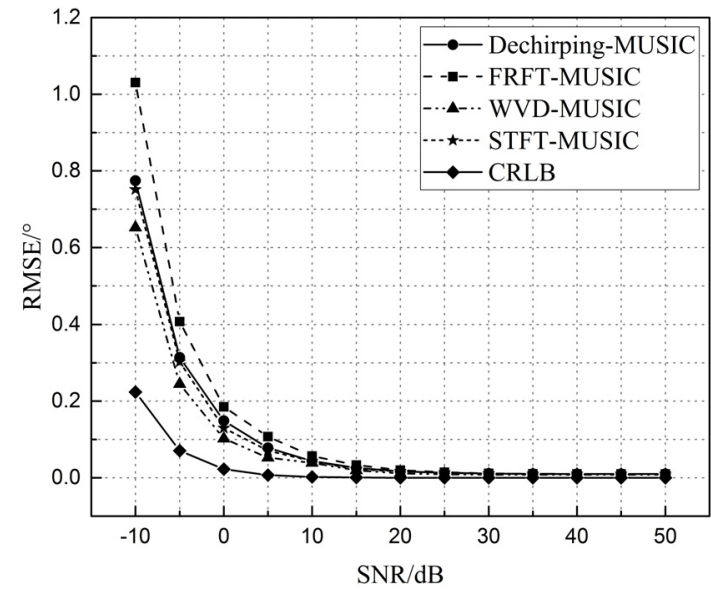

(b) Elevation angle based on MUSIC algorithm

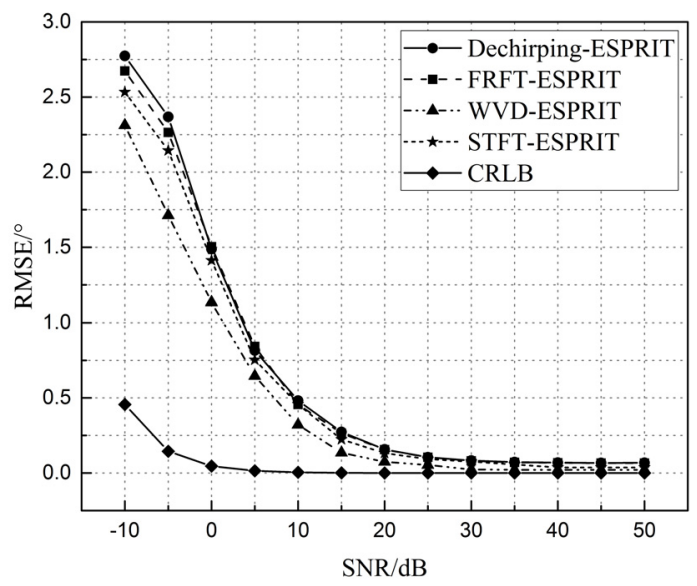

(c) Azimuth angle based on ESPRIT algorithm

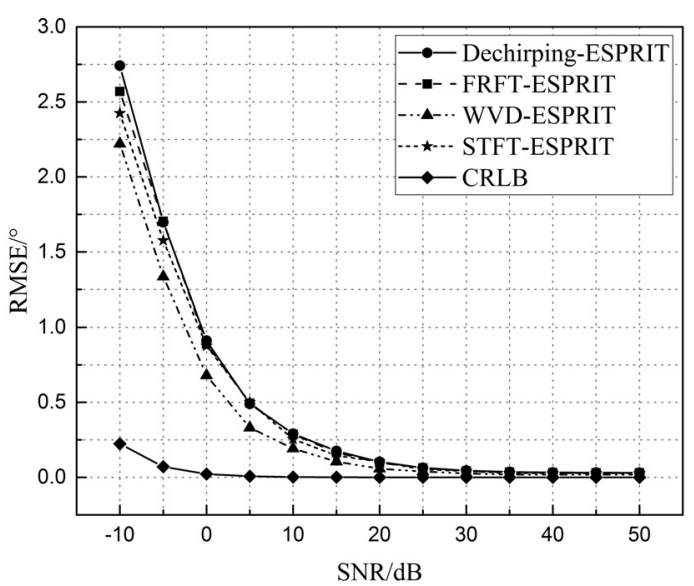

(d) Elevation angle based on ESPRIT algorithm

Fig. 6. RMSE of DOA estimation along with SNR using different algorithms.

So the proposed algorithm is prone to be easily realized in engineering.

In conclusion, we can contrast the main DOA estimation algorithm in many ways, such as the estimation precision, the computational complexity, the sampling rate, the cross-term interference and so on. The comparison results are shown in Tab. 3. 


\begin{tabular}{|c|c|c|c|}
\hline Algorithm & $\begin{array}{c}\text { Estimation } \\
\text { precision }\end{array}$ & $\begin{array}{c}\text { Computational } \\
\text { complexity }\end{array}$ & $\begin{array}{c}\text { Cross-term } \\
\text { interference }\end{array}$ \\
\hline Dechirping & $3 \mathrm{rd}$ & $O\left(M N L \log _{2} L\right)$ & $\mathrm{N}$ \\
\hline WVD & 1 st & $O\left(M N^{2} L^{2} \log _{2} L\right)$ & $\mathrm{Y}$ \\
\hline STFT & 2nd & $O\left(M N L^{2} \log _{2} L\right)$ & $\mathrm{N}$ \\
\hline FRFT & 4 th & $O\left(N L+M N^{2}\right)$ & $\mathrm{N}$ \\
\hline Algorithm & $\begin{array}{c}\text { Sampling } \\
\text { rate }\end{array}$ & $\begin{array}{c}\text { Time-frequency } \\
\text { point }\end{array}$ & $\begin{array}{c}\text { Window } \\
\text { function }\end{array}$ \\
\hline Dechirping & Normal & $\mathrm{N}$ & $\mathrm{N}$ \\
\hline WVD & High & $\mathrm{Y}$ & $\mathrm{N}$ \\
\hline STFT & Normal & $\mathrm{Y}$ & $\mathrm{Y}$ \\
\hline FRFT & Normal & $\mathrm{N}$ & $\mathrm{N}$ \\
\hline
\end{tabular}

Tab. 3. The comparison results of four algorithms.

In Tab. 3, "1st", "2nd", "3rd" and "4th" stand for the order of the estimation precision which means the algorithm based on WVD owns the highest estimation precision, followed by the algorithm based on STFT, our proposed algorithm and the algorithm based on FRFT. "N" stands for no and "Y" stands for yes. From Tab. 3, we can see that the proposed method owns high estimation precision and low computational complexity. Also it does not exist the cross-term interference and the puzzle of selecting the appropriate time-frequency point. The requirement for the system sampling is not high and it does not use the window function. The proposed algorithm also uses the relatively simple FFT operator. So it is easy to be realized in engineering.

\section{Conclusion}

In this paper, we proposed a new DOA estimation algorithm of multiple LFM signals based on the thoughts of Dechirping algorithm. Firstly, we proceed the difference frequency treatment between LFM signals received by each element and a specific reference signal. The reference signal is the signal to be estimated. It is received by the reference element. We can get a single-frequency pulse related to the signal to be estimated after Dechirping process. Then, we transform the Dechirping results from time domain to frequency domain by FFT to obtain an impulse pulse. We construct a new array data model through extracting the prominent parts of the impulse to obtain the time-invariant steering vector matrix. Finally, we realize 2-D DOA estimation of LFM signals by using MUSIC algorithm and ESPRIT algorithm respectively. The method proposed by this paper does not exist the cross-term interference and the puzzle of selecting the appropriate timefrequency point and window function. The computational complexity of the proposed algorithm achieves a low level contrast to the other DOA estimation algorithms based on time-frequency analysis tools. The algorithm also uses the relatively simple FFT operator and has the capability of estimating DOA of multiple LFM signals simultaneously with a high precision. The simulation results verify the effectiveness of the algorithm and it can be applied to DOA estimation of LFM signals.

\section{Appendix. The Derivation of (17)}

If there are $M$ LFM signals from far-field, according to (13), the output of the $n$th element can be written as:

$$
x_{n}=\left[\begin{array}{lll}
\exp \left(-\mathrm{j} 2 \pi f_{1} \tau_{n 1}\right) s_{1} & \cdots & \exp \left(-\mathrm{j} 2 \pi f_{M} \tau_{n M}\right) s_{M}
\end{array}\right] .
$$

We can carry out $N$ point discrete Fourier transform (DFT) on the output of the array element and get (27).

$$
\begin{aligned}
v_{q} & =\sum_{n=0}^{N-1} x_{n} \exp \left(-\mathrm{j} \frac{2 \pi}{N} n q\right) \\
& =\left[\begin{array}{l}
\sum_{n=0}^{N-1} \exp \left(-\mathrm{j} 2 \pi f_{1} \tau_{n 1}\right) \exp \left(-\mathrm{j} \frac{2 \pi}{N} n q\right) s_{1}, \ldots, \\
\sum_{n=0}^{N-1} \exp \left(-\mathrm{j} 2 \pi f_{M} \tau_{n M}\right) \exp \left(-\mathrm{j} \frac{2 \pi}{N} n q\right) s_{M}
\end{array}\right] \\
& \approx\left[N \mathrm{j}^{-q} J_{-q}\left(-\beta_{1}\right) \exp \left(-\mathrm{j} q \theta_{1}\right) s_{1}, \ldots, N \mathrm{j}^{-q} J_{-q}\left(-\beta_{M}\right) \exp \left(-\mathrm{j} q \theta_{M}\right) s_{M}\right] .
\end{aligned}
$$

In (27), $J_{m}(\cdot)$ stands for the order $m$ of Bessel function, $\beta_{m}$ is a factor related to the incoming wave parameters. $\beta_{m}=2 \pi \sin \left(\theta_{\mathrm{e} m}\right) r f_{m} / c, \beta=\max \left[2 \pi r f_{m} / c\right], q \in[-\lfloor\beta\rfloor,\lfloor\beta\rfloor] \cap Z$ and $L\rfloor$ is the rounded down symbol. $Z$ is the mathematical set of whole numbers. If $K=\lfloor\beta\rfloor$, the phase modes excited by this UCA [44] are: $-K,-K+1, \ldots, K$. So the number is $2 K+1$, which means the UCA can be equivalent to a ULA with $2 K+1$ elements.

$$
\text { If } u_{q}=v_{-q},(27) \text { can be expressed as matrix form: }
$$$$
\mathbf{U}=N \mathbf{J} \mathbf{A}_{c} \mathbf{S},
$$

$$
\mathbf{U}=\left[u_{-K}, \ldots, u_{K}\right]^{\mathrm{T}},
$$

$$
\mathbf{J}=\operatorname{diag}\left\{\mathrm{j}^{-K}, \ldots, \mathrm{j}^{K}\right\},
$$

$\mathbf{A}_{c}=\left[\begin{array}{ccc}J_{-K}\left(-\beta_{1}\right) \exp \left(-\mathrm{j} K \theta_{\mathrm{e} 1}\right) & \cdots & J_{-K}\left(-\beta_{M}\right) \exp \left(-\mathrm{j} K \theta_{\mathrm{e} M}\right) \\ \cdots & \ddots & \vdots \\ J_{K}\left(-\beta_{1}\right) \exp \left(\mathrm{j} K \theta_{\mathrm{e} 1}\right) & \cdots & J_{K}\left(-\beta_{M}\right) \exp \left(\mathrm{j} K \theta_{\mathrm{e} M}\right)\end{array}\right]$.

We can also express the definition of DFT as matrix form:

$$
\begin{gathered}
\mathbf{U}=\mathbf{F}^{\mathrm{H}} \mathbf{X}, \\
\mathbf{F}=\left[\begin{array}{llll}
\mathbf{w}_{-K} & \mathbf{w}_{-K+1} & \cdots & \mathbf{w}_{K}
\end{array}\right] \\
\mathbf{w}_{q}=\left[\begin{array}{llll}
1 & \exp \left(-\mathrm{j} \frac{2 \pi}{N} q\right) & \cdots & \left.\exp \left(-\mathrm{j} \frac{2 \pi(N-1)}{N} q\right)\right]^{\mathrm{H}} .
\end{array}\right.
\end{gathered}
$$

Based on (28) and (32), we can get:

$$
\mathbf{Y}=\mathbf{T X}=\mathbf{A}_{c} \mathbf{S} \text {. }
$$

In (35), $\mathbf{T}=\mathbf{J}^{-1} \mathbf{F}^{\mathrm{H}} / N$, which is called transformation matrix. In this way, we complete the mode-space transformation of UCA and the UCA with $N$ elements is equivalent to a ULA with $2 K+1$ elements. Then we can get (17). 


\section{References}

[1] XIAO, W., XIAO, X. C., TAI, H. M. Rank-1 ambiguity DOA estimation of circular array with fewer sensors. In Proceedings of the 45th IEEE Midwest Symposium on Circuits and Systems (MWSCAS-2002). Tulsa (USA), 2002, p. III-29 - III-32. DOI: 10.1109/MWSCAS.2002.1186962. ISBN: 0-7803-7523-8

[2] DU, W., SU, D. L., XIE, S. G., et al. A fast calculation method for the receiving mutual impedances of uniform circular arrays. IEEE Antennas and Wireless Propagation Letters, 2012, vol. 11, p. 893-896. DOI: 10.1109/LAWP.2012.2211329

[3] WANG, P., LI, Y. H., VUCETIC, B. Millimeter wave communications with symmetric uniform circular antenna arrays IEEE Communications Letters, 2014, vol. 18, no. 8, p. 1307-1310. DOI: 10.1109/LCOMM.2014.2332334

[4] DORSEY, W. M., COLEMAN, J. O., PICKLES, W. R. Uniform circular array pattern synthesis using second-order cone programming. IET Microwaves, Antennas \& Propagation, 2015, vol. 9, no. 8, p. 723-727. DOI: 10.1049/iet-map.2014.0418

[5] JACKSON, B. R., RAJAN, S., LIAO, B. J., et al. Direction of arrival estimation using directive antennas in uniform circular arrays. IEEE Transactions on Antennas and Propagation, 2015, vol. 63, no. 2, p. 736-747. DOI: 10.1109/TAP.2014.2384044

[6] WANG, M., MA, X. C., YAN, S. F., et al. An auto calibration algorithm for uniform circular array with unknown mutual coupling. IEEE Antennas and Wireless Propagation Letters, 2016, vol. 15 , p. 12-15. DOI: 10.1109/LAWP.2015.2425423

[7] PAN, Y. J., ZHANG, X. F., XIE, S. Y., et al. An ultra-fast DOA estimator with circular array interferometer using lookup table method. Radioengineering, 2015 , vol. 24 , no. 8 , p. $850-856$. DOI: $10.13164 /$ re.2015.0850

[8] JAIN, V., BLAIR, W. D. Filter design for steady-state tracking of maneuvering targets with LFM waveforms. IEEE Transactions on Aerospace and Electronic Systems, 2009, vol. 45, no. 2, p. 765-773. DOI: 10.1109/TAES.2009.5089558

[9] WANG, P., LI, H. B., DJUROVIC, P., et al. Integrated cubic phase function for linear FM signal analysis. IEEE Transactions on Aerospace and Electronic Systems, 2010, vol. 46, no. 3, p. 963-977. DOI: 10.1109/TAES.2010.5545167

[10] TAO, R., ZHANG, N., WANG, Y. Analyzing and compensating the effects of range and Doppler frequency migrations in linear frequency modulation pulse compression radar. IET Radar, Sonar and Navigation, 2011, vol. 5, no. 1, p. 12-22. DOI: 10.1049/ietrsn.2009.0265

[11] NGUYEN, V. K., TURLEY, M. D. E. Bandwidth extrapolation of LFM signals for narrowband radar systems. In International Conference on Radar. Adelaide (SA), 2013, vol. 51 no. 1, p. 702-712. DOI: 10.1109/RADAR.2013.6651975

[12] SU, J., TAO, H. H., RAO, X., et al. Coherently integrated cubic phase function for multiple LFM signals analysis. Electronics Letters, 2015, vol. 51 no. 5, p. 411-413. DOI: $10.1049 / \mathrm{el} .2014 .4164$

[13] YUAN, X. Direction-finding wideband linear FM sources with triangular arrays. IEEE Transactions on Aerospace and Electronic Systems, 2012, vol. 48, no. 3, p. 2416-2425. DOI: 10.1109/TAES.2012.6237600

[14] LAGHMARDI, N., HARABI, F., MEKNESSI, H., et al. A spacetime extended music estimation algorithm for wide band signals. Arabian Journal for Science and Engineering, 2013, vol. 38, no. 3, p. 661-667. DOI: 10.1007/s13369-012-0328-9

[15] SHA, Z. C., LIU, Z. M., HUANG, Z. T., et al. Covariance-based direction-of-arrival estimation of wideband coherent chirp signals via sparse representation. Sensors, 2013, vol. 13, no. 9, p. 11490-11497. DOI: $10.3390 / \mathrm{s} 130911490$

[16] HE, Z. Q., SHI, Z. P., HUANG, L., et al. Underdetermined DOA estimation for wideband signals using robust sparse covariance fitting. IEEE Signal Processing Letters, 2015, vol. 22, no. 4, p. 435-439. DOI: 10.1109/LSP.2014.2358084

[17] PAN, Y. J., TAI, N., YUAN, N. C. Wideband DOA estimation via sparse Bayesian learning over a Khatri-Rao dictionary. Radioengineering, 2015, vol. 24, no. 2, p. 552-557. DOI: $10.13164 /$ re. 2015.0552

[18] CHEN, H., WAN, Q., FAN, R., et al. Direction-of-arrival estimation based on sparse recovery with second-order statistics. Radioengineering, 2015, vol. 24, no. 1, p. 208-213. DOI: $10.13164 /$ re.2015.0208

[19] WANG, L., ZHAO, L. F., BI, G. A., et al. Novel wideband DOA estimation based on sparse Bayesian learning with Dirichlet process priors. IEEE Transactions on Signal Processing, 2016, vol. 64 , no. 2 , p. 275-289. DOI: 10.1109/TSP.2015.2481790

[20] AMIN, M. G. Spatial time frequency distributions for direction finding and blind source separation. In The International Society for Optical Engineering (Proc Spie). Orlando (USA), 1999, vol. 3723 , p. $62-70$. DOI: $10.1117 / 12.342958$

[21] BELOUCHRANI, A., AMIN, M. G. Time frequency MUSIC. IEEE Signal Processing Letters, 1999, vol. 6, no. 5, p. 109-110. DOI: $10.1109 / 97.755429$

[22] ZHANG, Y. M. D., AMIN, M. G., HIMED, B. Joint DOD/DOA estimation in MIMO radar exploiting time-frequency signal representations. EURASIP Journal on Advances in Signal Processing, 2012, vol. 2012, no. 1, p. 1-10. DOI: 10.1186/16876180-2012-102

[23] KHODJA, M., BELOUCHRANI, A., ABED-MERAIM, K. Performance analysis for time-frequency MUSIC algorithm in presence of both additive noise and array calibration errors. EURASIP Journal on Advances in Signal Processing, 2012, vol. 2012, no. 1, p. 1-11. DOI: 10.1186/1687-6180-2012-94

[24] LIN, J. C., MA, X. C., YAN, S. F., et al. Time-frequency multiinvariance esprit for DOA estimation. IEEE Antennas and Wireless Propagation Letters, 2015, vol. 15, p. 770-773. DOI: 10.1109/LAWP.2015.2473664

[25] LI, L. P., HUANG, K. J., CHEN, T. Q. 2-D DOA estimation of coherent wideband FM signals based on STFT. Journal of Electronics and Information Technology, 2005, vol. 27, no. 11, p. 1760-1764. (in Chinese)

[26] ZHANG, H. J., BI, G. A., CAI, Y. L., et al. DOA estimation of closely-spaced and spectrally-overlapped sources using a STFTbased MUSIC algorithm. Digital Signal Processing, 2016, vol. 52, p. 25-34. DOI: 10.1016/j.dsp.2016.01.015

[27] YANG, X. M., TAO, R. 2D DOA estimation of LFM signals based on fractional Fourier transform and ESPRIT algorithm. Acta Armamentarii, 2007, vol. 28, no. 12, p. 1438-1442. (in Chinese)

[28] YANG, X. M., TAO, R. 2-D DOA estimation of LFM signals based on fractional Fourier transform. Acta Electronica Sinica, 2008, vol. 36, no. 9, p. 1737-1740. (in Chinese)

[29] YANG, W., SHI, Y. W. FRFT based method to estimate DOA for wideband signal. Advanced Materials Research, 2013, vol. 712-715, p. 2716-2720.

[30] CUI, Y., WANG, J., F. Wideband LFM interference suppression based on fractional Fourier transform and projection techniques. Circuits Systems and Signal Process, 2014, vol. 33, no. 2, p. 613-627. DOI: $10.1007 / \mathrm{s} 00034-013-9642-\mathrm{Z}$

[31] YU, J. X., ZHANG, L., LIU, K. H., et al. Separation and localization of multiple distributed wideband chirps using the 
fractional Fourier transform. EURASIP Journal on Wireless Communications and Networking, 2015, vol. 266, p. 1-8. DOI: 10.1186/s13638-015-0497-9

[32] YU, J. X., ZHANG, L., LIU, K. H. Coherently distributed wideband LFM source localization. IEEE Signal Processing Letters, 2015, vol. 22, no. 4, p. 504-508. DOI: 10.1109/LSP.2014.2363843

[33] TANG, X. H., LI, Q. L. Time Frequency Analysis and Wavelet Transform. Beijing (China): Science Press, 2016. ISBN: 978-7-03047542-8. (in Chinese)

[34] BAO, Z., XING, M. D., WANG, T. Radar Imaging Technology. Beijing (China): Publishing of Electronics Industry, 2014. ISBN: 978-7-121-01072-9. (in Chinese)

[35] SCHMIDT, R. O. Multiple emitter location and signal parameter estimation. IEEE Transactions on Antennas and Propagation, 1986, vol. 34, no. 3, p. 276-280. DOI: 10.1109/TAP. 1986.1143830

[36] ROY, R., PAULRAJ, A., KAILATH, T. ESPRIT-a subspace rotation approach to estimation of parameters of cissoids in noise. IEEE Transactions on Acoustics, Speech, and Signal Processing, 1986, vol. 34 , no. 5, p. $1340-1342$. DOI: 10.1109/TASSP.1986.1164935

[37] ROY, R., KAILATH, T. ESPRIT-estimation of signal parameters via rotational invariance techniques. IEEE Transactions on Acoustics, Speech, and Signal Processing, 1989, vol. 37, no. 7, p. 984-995. DOI: $10.1109 / 29.32276$

[38] GRIFFITHS, H. D., EIGES, R. Sectoral phase modes from circular antenna arrays. Electronics Letters, 1992, vol. 28, no. 17, p. 1581-1582. DOI: 10.1049/el:19921006

[39] MATHEWS, C. P., ZOLTOWSKI, M. D. Eigenstructrure techniques for 2-D angle estimation with uniform circular arrays. IEEE Transactions on Signal Processing, 1994, vol. 42, no. 9, p. 2395-2407. DOI: $10.1109 / 78.317861$

[40] TOMIC, S., BEKO, M., DINIS, R. RSS-based localization in wireless sensor networks using convex relaxation: noncooperative and cooperative schemes. IEEE Transactions on Vehicular Technology, 2015, vol. 64, no. 5, p. 2037-2050. DOI: 10.1109/TVT.2014.2334397

[41] TOMIC, S., BEKO, M., DINIS, R. Distributed RSS-AoA based localization with unknown transmit powers. IEEE Wireless Communications Letters, 2016, vol. 5, no. 4, p. 392-395. DOI: 10.1109/LWC.2016.2567394

[42] TOMIC, S., BEKO, M., DINIS, R., et al. A closed-form solution for RSS/AoA target localization by spherical coordinates conversion. IEEE Wireless Communications Letters, 2016, vol. 5, no. 6, p. 680-683. DOI: 10.1109/LWC.2016.2615614

[43] TOMIC, S., BEKO, M., DINIS, R., et al. Distributed algorithm for target localization in wireless sensor networks using RSS and AoA measurements. Pervasive and Mobile Computing, 2016. DOI: 10.1016/j.pmcj.2016.09.013
[44] WANG, Y. L., CHEN, H., PENG, Y. N., et al. Spatial Spectrum Estimation. Beijing (China): Tsinghua University Press, 2004. ISBN: 7-302-09209-5. (in Chinese)

\section{About the Authors ...}

Kaibo CUI was born in 1990. He received his M.S. degree in Electronic Science and Technology from the National University of Defense Technology in 2013. Currently he is working towards the Ph.D. degree in the College of Electronic Science and Engineering, National University of Defense Technology, Changsha, Hunan, China. His research interests include signal processing and spatial spectrum estimation.

Weiwei WU was born in 1981. She received her M.S. and Ph.D. degree in Electronic Science and Technology from the National University of Defense Technology in 2008 and 2011, respectively. Currently she is a teacher in the College of Electronic Science and Engineering, National University of Defense Technology, Changsha, Hunan, China. Her research interests include array signal processing and antenna design.

Xi CHEN was born in 1983. He received his Ph.D. degree in Electronic Science and Technology from the National University of Defense Technology in 2013. Currently he is a teacher in the College of Electronic Science and Engineering, National University of Defense Technology, Changsha, Hunan, China. His research interests include signal processing.

Jingjian HUANG was born in 1983. He received his Ph.D. degree in Electronic Science and Technology from the National University of Defense Technology in 2014. Currently he is a teacher in the College of Electronic Science and Engineering, National University of Defense Technology, Changsha, Hunan, China. His research interests include antenna design.

Naichang YUAN was born in 1965. He received his M.S. and $\mathrm{Ph} . \mathrm{D}$. degree in Electronic Science and Technology from the University of Science and Technology of China in 1991 and 1994, respectively. He is currently a professor with the College of Electronic Science and Engineering, National University of Defense Technology, Changsha, Hunan, China. His research interests include array signal processing, signal processing in radar. 